hep-ph/9908079

\title{
Relativistic Modification of the Gamow Factor
}

\author{
Jin-Hee Yoon ${ }^{1,2}$ and Cheuk-Yin Wong $^{2}$ \\ ${ }^{1}$ Dept. of Physics, Inha University, Inchon, South Korea \\ ${ }^{2}$ Physics Division, Oak Ridge National Laboratory, Oak Ridge, Tennessee, U.S.A.
}

(August 29, 2018)

\begin{abstract}
In processes involving Coulomb-type initial- and final-state interactions, the Gamow factor has been traditionally used to take into account these additional interactions. The Gamow factor needs to be modified when the magnitude of the effective coupling constant increases or when the velocity increases. For the production of a pair of particles under their mutual Coulomb-type interaction, we obtain the modification of the Gamow factor in terms of the overlap of the Feynman amplitude with the relativistic wave function of the two particles. As a first example, we study the modification of the Gamow factor for the production of two bosons. The modification is substantial when the coupling constant is large.
\end{abstract}

\section{INTRODUCTION}

Initial- and final- state interactions are important in many branches of theoretical physics involving the reaction or the production of particles. They have a great influence on the reaction rates or the production cross sections [1-11]. These initial- and final-state interactions lead to a large enhancement of the cross section if the particles are subject to a strong attractive interaction; they can lead to a large suppression under a strong repulsive interaction. We shall use the term "the $K$-factor" to label the ratio of the cross section with the interaction to the corresponding quantity without the interaction.

As is well known, for interactions such as the electric-Coulomb and color-Coulomb interaction $V(r)=-\alpha / r$ in non-relativistic physics, the effect of the initial- or final-state interactions leads to a $K$-factor given by the Gamow-Sommerfeld factor [2,3]. The GamowSommerfeld factor (or simply called, the Gamow factor) is given explicitly by

$$
G(\eta)=\frac{2 \pi \eta}{1-e^{-2 \pi \eta}}
$$

where

$$
\eta=\frac{\alpha}{v} .
$$


The magnitude of the relative velocity $v$ is the ratio of the asymptotic momentum $p$ to the energy $\epsilon_{w}$ in the relative coordinate system (see Eqs. (13) and (18) below). Following Todorov [12], Crater and Van Alstine [13], and Eqs. (21.13a)-(21.13c) of Crater et al. [14], the relative velocity for the particles $a$ and $b$ is related to their center-of-mass energy $\sqrt{s}$ by

$$
v=\frac{\left(s^{2}-4 s m^{2}\right)^{1 / 2}}{s-2 m^{2}} .
$$

This gives $v \sim 2 \sqrt{1-4 m^{2} / s}$ when $\sqrt{s} \sim 2 m$ and $v \rightarrow 1$ when $s \rightarrow \infty$. This Gamow factor has been used to study initial- and final-state interactions in reaction processes.

There are physical processes in which the coupling constant of the interaction between the particles can be quite large and the use of the Gamow factor to correct the initialstate and final-state interactions may not be adequate. For example, in the annihilation or the production of $q \bar{q}$ pairs, the interaction arising from the exchange of a gluon leads to a color-Coulomb interaction with a coupling constant $\alpha$ about $0.2-0.4$, depending on the renormalization scale of the reaction process. Another example of strong coupling occurs in the case of a negatively charged particle in a nucleus with a large $Z$ number. Such a large coupling constant will also lead to a modification of the Gamow factor as there are higher-order effects of the potential which are important when the coupling constant becomes large. One can mention, for example, the well-known case of the "Landau fall" which is the relativistic nonperturbative collapse of the wave function for an attractive Coulomb-type potential when the coupling constant exceeds a certain limit [15]. Furthermore, although the effect of the interaction is very large for low relative velocities, it is useful to see how the effect varies as the velocity increases.

While one sees the need to use the relativistic formalism to study the case with high relative velocities, one may wonder what special cases can be of interest to use a relativistic formalism for the case of low relative velocities. By the term "the relative velocity", we usually refer to the relative velocity between the particles in the asymptotic region of $r \rightarrow \infty$ where there is no interaction. However, when there is a strongly attractive interaction, the actual relative velocity depends on the spatial location. One can envisage that if the coupling constant is large, the motion of the two particles at small distances can become relativistic, even though the relative velocity at $r \rightarrow \infty$ is small. Hence, it is necessary to use the relativistic formalism to study the effects of the mutual interaction with large coupling constants, even for the case of low asymptotic relative velocities at $r \rightarrow \infty$.

The $K$-factor for the Coulomb potential can be studied by examining the two-body wave function in the Klein-Gordon or the Dirac equation involving a Coulomb potential. Compared with the non-relativistic Schrödinger equation involving the Coulomb potential, there is an additional effective attractive potential, $-|V(r)|^{2} / 2 m_{w}$ (see Eq. (16) below), which leads to a non-trivial behavior when the coupling constant becomes large. In the case of fermions under the Coulomb interaction, there are further modifications associated with additional spin-dependent potential terms.

The question of initial- and final-state interactions is also related to the question of the decay and the production of bound states when the interactions lead to the formation of bound states [16, 17]. Previously, the decay of the bound positronium ${ }^{1} S_{0}$ state into two photons has been studied in the relativistic formalism by Crater [17]. In our present study, we are interested mainly in the case of two particles in the continuum. We wish to find out 
how the mutual interaction may affect their reaction or production rates.

The standard method of calculating the $K$-factor is by evaluating the absolute square of the wave function at the origin. Such a method breaks down for the relativistic case as the wave function is infinite at the origin [18. The proper method to obtain the $K$-factor is by taking the overlap of the relativistic wave function with the Feynman amplitude. As an illustration, we shall try out the method for the production of a pair of scalar particles interacting with a Coulomb-type final-state interaction.

\section{THE $K$-FACTOR}

The effects of the final- and initial-state interaction depend on the physical process. Here we shall be interested in the class of processes involving the reaction or the production of a pair of particles $a$ and $b$, subject to the mutual interaction between $a$ and $b$. For definiteness, we shall study the production process, as the $K$-factor is the same for production or reaction.

The simplest description of such a process is in terms of the perturbation theory which gives the amplitude for the production of this pair of particles $a$ and $b$. The state $\Phi_{a b}$ of the $a b$ pair after the reaction $x+y \rightarrow a+b$ is represented by the state vector

$$
\left|\Phi_{a b}\right\rangle=\mathcal{M}(x y \rightarrow a(P / 2+q) b(P / 2-q))|a(P / 2+q) b(P / 2-q)\rangle
$$

where $\mathcal{M}(x y \rightarrow a b)$ is the Feynman amplitude for the $x+y \rightarrow a+b$ process. For the two-particle system $a b$, we define the center-of-mass momentum $P=a+b$ and the relative momentum $q=(a-b) / 2$.

On the other hand, under their mutual interaction which can be represented by a twobody potential $V(r)$ between $a$ and $b$, we can describe an $a b$ pair with a center-of-mass momentum $P$ as

$$
\left|\Psi_{V}\right\rangle=\tilde{\psi}(q)|P\rangle .
$$

The probability amplitude for the production of an $a b$ pair under their mutual interaction is obtained by taking the overlap of the amplitude in (4) with the wave function in (5). The overlap is the simplest in the $a b$ center-of-mass system where $P=(\sqrt{s}, \mathbf{0})$ and $q=(0, \mathbf{q})$ and the (unnromalized) probability amplitude is [16,17, 19.

$$
\left\langle\Psi_{V} \mid \Phi_{a b}\right\rangle=\int \frac{d^{3} \mathbf{q}}{(2 \pi)^{3}} \tilde{\psi}(\mathbf{q}) \mathcal{M}(x y \rightarrow a(\boldsymbol{q}) b(-\boldsymbol{q})) .
$$

The $K$-factor for the occurrence of $a b$ in the state $\Psi_{V}$ is then given by

$$
K \equiv \frac{\left|\left\langle\Psi_{V} \mid \Phi_{a b}\right\rangle\right|^{2}}{\left|\left\langle\Psi_{0} \mid \Phi_{a b}\right\rangle\right|^{2}}=\frac{\text { (Production cross section with final-state interaction) }}{\text { (Production cross section without final-state interaction) }}
$$

where $\left|\Psi_{0}\right\rangle$ is the state of the $a b$ pair without their mutual interaction. We have used the unnormalized amplitude in Eq. (6) as any normalizsation constant will cancel out in the definition of the $K$-factor in Eq. (7). The result of the cross section calculated using the simple first-order diagram can be corrected to include the effects of the final-state interaction by multiplying with the $K$-factor:

$$
\left(\begin{array}{c}
\text { Production cross section } \\
\text { with final-state interaction }
\end{array}\right)=K \times\left(\begin{array}{c}
\text { Production cross section } \\
\text { without final-state interaction }
\end{array}\right) \text {. }
$$




\section{KLEIN-GORDON EQUATION FOR THE COULOMB INTERACTION}

In this first study, in order to illustrate the main features of the effect and to avoid complications brought on by the spinor algebra, we shall carry out the procedures outlined above for the production of two scalar particles.

We need to separate out the center-of-mass motion and the relative motion for these two particles. Consider first the case without a mutual interaction as in the region where the two particles are far apart. The two particles have 4-momenta $p_{1}$ and $p_{2}$ and rest masses $m_{1}$ and $m_{2}$. We introduce the total momentum $P$ [13, 14

$$
P=p_{1}+p_{2}
$$

and the relative momentum $q$

$$
q=w_{1} p_{1}-w_{2} p_{2}
$$

where

$$
w_{1}=\frac{s-m_{2}^{2}+m_{1}^{2}}{2 s}
$$

and

$$
w_{2}=\frac{s-m_{1}^{2}+m_{2}^{2}}{2 s}
$$

with $s=P^{2}$. We have the following identity

$$
p_{1}^{2}-m_{1}^{2}+p_{2}^{2}-m_{2}^{2}=\left(w_{1}^{2}+w_{2}^{2}\right) P^{2}+2 q^{2}-m_{1}^{2}-m_{2}^{2}=0 .
$$

We can choose to work in the center-of-mass system in which $P=(\sqrt{s}, \mathbf{0})$ and $q=(0, \boldsymbol{q})$. The above equation can be written in terms of an effective energy $\epsilon_{w}$, and a generalized reduced mass $m_{w}$ as

$$
\epsilon_{w}^{2}-\boldsymbol{q}^{2}-m_{w}^{2}=0
$$

where

$$
\epsilon_{w}=\frac{s-m_{1}^{2}-m_{2}^{2}}{2 \sqrt{s}}
$$

and

$$
m_{w}=\frac{m_{1} m_{2}}{\sqrt{s}}
$$

Next, we study the system of masses $m_{1}=m_{2}=m$ interacting with a mutual Coulombtype interaction

$$
V(r)=-\frac{\alpha}{r}
$$

The equation of motion can be obtained from Eq. (12) by the canonical method of replacing $\epsilon_{w}$ with $\epsilon_{w}-V(r)$. The Klein-Gordon equation for the two-particle system under a mutual Coulomb-type interaction $V(r)$ is 


$$
\left\{\left[\epsilon_{w}-V(r)\right]^{2}-\boldsymbol{q}^{2}-m_{w}^{2}\right\} \psi(\boldsymbol{r})=0
$$

Writing $\psi(\boldsymbol{r})=R_{n l}(r) Y_{l m}(\theta, \phi)$ in (16), the equation for $R_{n l}(r)$ is

$$
\left[\frac{d}{d z^{2}}+\frac{2}{z} \frac{d}{d z}-\frac{l(l+1)}{z^{2}}+\frac{2 \eta}{z}+\frac{\alpha^{2}}{z^{2}}+1\right] R_{n l}(r)=0
$$

where $z=p r, p$ is the asymptotic momentum at $r \rightarrow \infty$ given by

$$
p=\sqrt{\epsilon_{w}^{2}-m_{w}^{2}} \text {. }
$$

The wave function $R_{n l}(r)$ can be represented by the dimensionless variable $z=p r$ and is characterized by two dimensionless parameters: $\eta=\alpha / v$ and $\alpha^{2}$, where $v=p / \epsilon_{w}$ is given by Eq. (3).

The solution of Eq. (17) is

$$
R_{n l}(r)=\frac{|\Gamma(a)|}{\Gamma(b)} e^{\pi \eta / 2}(2 i z)^{\mu-1 / 2} e^{-i z}{ }_{1} F_{1}(a, b, 2 i z)
$$

where

$$
\begin{gathered}
a=\mu+\frac{1}{2}+i \eta, \\
b=2 \mu+1, \\
\mu=\sqrt{\left(l+\frac{1}{2}\right)^{2}-\alpha^{2},}
\end{gathered}
$$

${ }_{1} F_{1}$ is the confluent hypergeometric function, and the normalization constant has been determined by using the boundary condition that at $r \rightarrow \infty, R_{n l}(r) \rightarrow \sin \left(p r+\delta_{l}\right) / p r$ with the Coulomb phase shift $\delta_{l}$. For the S-state, the critical value of $\alpha$ is $1 / 2$.

\section{THE FEYNMAN AMPLITUDE AND THE OVERLAP WITH THE COULOMB WAVE FUNCTION}

To obtain the effect of the final-state interaction between $a$ and $b$ produced in a pairproduction process, we consider the production of the pair of bosons from the fusion of two photons. Because the relevant factors associated with the mode of production will be cancelled out at the end in Eq. (7), the results of the $K$-factor depend only on the final-state interaction and is the same for a similar mode of production of the pair of bosons.
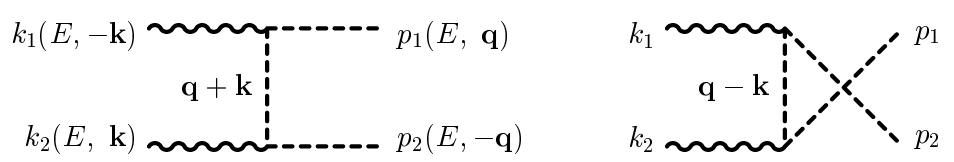

Fig. 1. Feynman diagrams included in the calculation. 
The diagrams we include are shown in Fig. 1 which give the amplitude

$$
-i \mathcal{M}(x y \rightarrow a(P / 2+q) b(P / 2-q))=i e^{2}\left[-\frac{(2 \boldsymbol{q}+\boldsymbol{k}) \cdot \boldsymbol{\epsilon}_{1} \boldsymbol{k} \cdot \boldsymbol{\epsilon}_{2}}{(\boldsymbol{q}+\boldsymbol{k})^{2}+m^{2}}+\frac{(2 \boldsymbol{q}-\boldsymbol{k}) \cdot \boldsymbol{\epsilon}_{2} \boldsymbol{k} \cdot \boldsymbol{\epsilon}_{1}}{(\boldsymbol{q}-\boldsymbol{k})^{2}+m^{2}}\right]
$$

where $\boldsymbol{k}$ is the momentum of the photon, $\boldsymbol{q}$ is the momentum of one of the bosons, and $\epsilon_{i}$ is the polarization vector of the $i$-th photon. The overlap of the wave function with the Feynman amplitude is then

$$
\left\langle\Psi_{V} \mid \Phi_{a b}\right\rangle=i e^{2} \int \frac{d \boldsymbol{q}}{(2 \pi)^{3}} \tilde{\psi}(\boldsymbol{q})\left[-\frac{(2 \boldsymbol{q}+\boldsymbol{k}) \cdot \boldsymbol{\epsilon}_{1} \boldsymbol{k} \cdot \boldsymbol{\epsilon}_{2}}{(\boldsymbol{q}+\boldsymbol{k})^{2}+m^{2}}+\frac{(2 \boldsymbol{q}-\boldsymbol{k}) \cdot \boldsymbol{\epsilon}_{2} \boldsymbol{k} \cdot \boldsymbol{\epsilon}_{1}}{(\boldsymbol{q}-\boldsymbol{k})^{2}+m^{2}}\right] .
$$

As the Coulomb wave function of Eq. (19) is given in the configuration space, it is useful to write the above integral in terms of the wave function in configuration space. The latter is given by

$$
\psi(\boldsymbol{r})=\int \frac{d \boldsymbol{q}}{(2 \pi)^{3}} \tilde{\psi}(\boldsymbol{q}) e^{-i \boldsymbol{q} \cdot \boldsymbol{r}}
$$

In conventional applications, one expands the Feynman amplitude (23) in powers of $\boldsymbol{q}$ and keeps only the lowest order $q$-independent term $\mathcal{M}_{0}$ :

$$
\mathcal{M} \approx \mathcal{M}_{0}+O(|\boldsymbol{q}|)
$$

In this approximation of taking only the leading term, Eqs. (7) and (25) then give the usual $K$-factor as the absolute square of the wave function $\psi(r)$ at the origin

$$
K=|\psi(r=0)|^{2} .
$$

However, such an approximation cannot be applied to our case with the relativistic wave function as the wave function, Eq. (19), is infinite at the origin. To avoid this singular behavior, the full Feynman amplitude is needed to evaluate the overlap integral and the $K$-factor in Eqs. (23) and (7).

In terms of the spatial wave function, the overlap integral (23) is

$$
\begin{aligned}
\left\langle\Psi_{V} \mid \Phi_{a b}\right\rangle=e^{2} \int d \boldsymbol{r} \psi(\boldsymbol{r})[ & e^{-i \boldsymbol{k} \cdot \boldsymbol{r}}\left\{2 \hat{\boldsymbol{\epsilon}}_{1} \cdot \frac{\nabla}{i}-\hat{\boldsymbol{\epsilon}}_{1} \cdot \boldsymbol{k}\right\} \frac{e^{-m r}}{4 \pi r} \hat{\boldsymbol{\epsilon}}_{2} \cdot \boldsymbol{k} \\
& \left.-e^{i \boldsymbol{k} \cdot \boldsymbol{r}}\left\{2 \hat{\boldsymbol{\epsilon}}_{2} \cdot \frac{\nabla}{i}+\hat{\boldsymbol{\epsilon}}_{2} \cdot \boldsymbol{k}\right\} \frac{e^{-m r}}{4 \pi r} \hat{\boldsymbol{\epsilon}}_{1} \cdot \boldsymbol{k}\right] .
\end{aligned}
$$

We shall specialize to the S-wave case with $l=0$. Using the wave function of Eq. (19), we carry out the above integration and obtain

$$
\begin{aligned}
\left\langle\Psi_{V}\right| & \left.\Phi_{a b}\right\rangle=2 e^{2}\left(\hat{\boldsymbol{\epsilon}}_{1} \cdot \boldsymbol{k}\right)\left(\hat{\boldsymbol{\epsilon}}_{2} \cdot \boldsymbol{k}\right) \frac{|\Gamma(a)|}{\Gamma(b)} e^{\pi \eta / 2} \sum_{n=0}^{\infty} \frac{(a)_{n} \Gamma\left(\frac{3}{2}+\mu+n\right)}{(b)_{n} n !}\left(\frac{2 i p}{\sqrt{\delta^{2}+k^{2}}}\right)^{n+\mu-\frac{1}{2}} \frac{1}{\delta^{2}+k^{2}} \\
\times & {\left[\frac{2}{3} F\left(\frac{3}{4}+\frac{\mu+n}{2}, \frac{5}{4}-\frac{\mu+n}{2} ; \frac{5}{2} ; \xi^{2}\right)+\frac{2 m}{3} \frac{\frac{3}{2}+\mu+n}{\sqrt{\delta^{2}+k^{2}}} F\left(\frac{5}{4}+\frac{\mu+n}{2}, \frac{3}{4}-\frac{\mu+n}{2} ; \frac{5}{2} ; \xi^{2}\right)\right.} \\
& \left.-F\left(\frac{3}{4}+\frac{\mu+n}{2}, \frac{1}{4}-\frac{\mu+n}{2} ; \frac{3}{2} ; \xi^{2}\right)\right],
\end{aligned}
$$


where

$$
\begin{gathered}
\delta=m+i p \\
\xi^{2}=\frac{k^{2}}{(m+i p)^{2}+k^{2}} .
\end{gathered}
$$

In Eq. (29) $(a)_{n}=a(a+1)(a+2) . .(a+n-1)$, with $(a)_{0}=1$. The quantity $(b)_{n}$ is similarly defined.

\section{RESULTS FOR THE $K$-FACTOR}

We introduce the complex angle variable

$$
\theta=\tan ^{-1} \frac{k}{m+i p}=\frac{\pi}{4}-i \frac{1}{4} \ln \frac{k+p}{k-p}
$$

which is a relativistic measure of the relative motion between particles $a$ and $b$. The real part of $\theta$ is always $\pi / 4$, and the imaginary part is negative, with a magnitude that is half of the rapidity of the produced particle in the center-of-mass system.

In terms of the angle variable, Eq.(29) can be transformed as follows:

$$
\left\langle\Psi_{V} \mid \Phi_{a b}\right\rangle=2 e^{2}\left(\hat{\boldsymbol{\epsilon}}_{1} \cdot \boldsymbol{k}\right)\left(\hat{\boldsymbol{\epsilon}}_{2} \cdot \boldsymbol{k}\right) \frac{|\Gamma(a)|}{\Gamma(b)} e^{\pi \eta / 2} \mathcal{A},
$$

where the factor $\mathcal{A}$ is

$$
\begin{aligned}
\mathcal{A} & =\sum_{n=0}^{\infty} \frac{(a)_{n} \Gamma(2 \nu)}{(b)_{n} n !}\left(\frac{2 i p}{\sqrt{\delta^{2}+k^{2}}}\right)^{n+\mu-\frac{1}{2}} \frac{1}{k^{2} \sin \theta} \\
& \times\left[\frac{2}{2 \nu-3}\left\{\frac{\sin (2 \nu-2) \theta}{2 \nu-2} \cos \theta-\frac{\sin (2 \nu-1) \theta}{2 \nu-1}\right\}\right. \\
& \left.+\frac{2 m}{k} \frac{2 \nu}{2 \nu-2} \sin \theta\left\{\frac{\sin (2 \nu-1) \theta}{2 \nu-1} \cos \theta-\frac{\sin 2 \nu \theta}{2 \nu}\right\}-\sin ^{2} \theta \frac{\sin (2 \nu-1) \theta}{2 \nu-1}\right],
\end{aligned}
$$

and $\nu=3 / 4+(\mu+n) / 2$. To obtain the $K$-factor, we also need the overlap between the Feynman amplitude and the wave function without the final-state interaction. By using the wave function $\psi_{0}(r)=\sin p r / p r$ for the S-state without the Coulomb potential, we obtained the amplitude for the case without the final-state interaction as given by

$$
\left\langle\Psi_{0} \mid \Phi_{a b}\right\rangle=2 e^{2}\left(\hat{\boldsymbol{\epsilon}}_{1} \cdot \boldsymbol{k}\right)\left(\hat{\boldsymbol{\epsilon}}_{2} \cdot \boldsymbol{k}\right) \mathcal{B}
$$

where the factor $\mathcal{B}$ is

$$
\mathcal{B}=\mathcal{I} m\left\{\left(\cot \theta^{*}-2 m / k\right)\left(\theta^{*} \cot \theta^{*}-1\right)\right\} / p k,
$$

and $\theta^{*}$ is a complex conjugate of $\theta$. Then the ratio between the absolute squares of Eqs.(33) and (35) is the relativistic expression of the $K$-factor, 


$$
K=\left|\frac{\Gamma(a)}{\Gamma(b)} e^{\pi \eta / 2} \frac{\mathcal{A}}{\mathcal{B}}\right|^{2}
$$

We can identify the factor $\left|\Gamma(a) e^{\pi \eta / 2} / \Gamma(b)\right|^{2}$ as closely related to the Gamow factor $G(\eta)$. One can show that

$$
\left|\frac{\Gamma(a)}{\Gamma(b)} e^{\pi \eta / 2}\right|^{2}=G(\eta)\left|\frac{\Gamma(\mu+1 / 2)}{\Gamma(2 \mu+1)} \prod_{j=0}^{\infty}\left(1+\frac{\mu-1 / 2}{1+j}\right)^{2}\left(1+\frac{\left(\frac{3}{2}+\mu+2 j\right)\left(\frac{1}{2}-\mu\right)}{\left(\mu+\frac{1}{2}+j\right)^{2}+\eta^{2}}\right)\right|^{2} .
$$

Therefore, the proper treatment of the dynamics of the interacting particles leads to the modification of the Gamow factor $G(\eta)$ of Eq. (1) by a factor $\kappa$ given by

$$
K=G(\eta) \kappa
$$

where

$$
\kappa=\left|\frac{\Gamma(\mu+1 / 2)}{\Gamma(2 \mu+1)} \prod_{j=0}^{\infty}\left(1+\frac{\mu-1 / 2}{1+j}\right)^{2}\left(1+\frac{\left(\frac{3}{2}+\mu+2 j\right)\left(\frac{1}{2}-\mu\right)}{\left(\mu+\frac{1}{2}+j\right)^{2}+\eta^{2}}\right)\right|^{2}\left|\frac{\mathcal{A}}{\mathcal{B}}\right|^{2},
$$

and

$$
\begin{aligned}
\left|\frac{\mathcal{A}}{\mathcal{B}}\right|^{2}= & \mid \sum_{n=0}^{\infty} \frac{(a)_{n} \Gamma(2 \nu)}{(b)_{n} n ! \sin \theta}\left(\frac{2 i p}{\sqrt{\delta^{2}+k^{2}}}\right)^{n+\mu-\frac{1}{2}}\left[\frac{2}{2 \nu-3}\left\{\frac{\sin (2 \nu-2) \theta}{2 \nu-2} \cos \theta-\frac{\sin (2 \nu-1) \theta}{2 \nu-1}\right\}\right. \\
& \left.+\frac{2 m}{k} \frac{2 \nu}{2 \nu-2} \sin \theta\left\{\frac{\sin (2 \nu-1) \theta}{2 \nu-1} \cos \theta-\frac{\sin 2 \nu \theta}{2 \nu}\right\}-\sin ^{2} \theta \frac{\sin (2 \nu-1) \theta}{2 \nu-1}\right]\left.\right|^{2} \\
& /\left|(k / p) \mathcal{I} m\left\{\left(k \cot \theta^{*}-2 m\right)\left(\theta^{*} \cot \theta^{*}-1\right)\right\}\right|^{2} .
\end{aligned}
$$

In the limit of $\alpha \rightarrow 0$ or $v \rightarrow 0$, the factor $\kappa$ goes to 1 and is consistent with the Gamow factor.

We note that the center-of-mass energy $\sqrt{s}$ in units of the rest mass of the produced particle is a function of $\eta / \alpha$ :

$$
\frac{\sqrt{s}}{m}=\sqrt{2\left(1+\frac{\eta / \alpha}{\sqrt{\eta^{2} / \alpha^{2}-1}}\right)}
$$

Various other kinematic variables, such as $k / m=\sqrt{s} / 2 m$ and $p / m=\sqrt{s / 4 m^{2}-1}$ can be similarly expressed as a function of $\eta / \alpha$. From these relations and the relation between the $K$-factor and $\eta$ and $\alpha$, we can study the $K$-factor for the production of a pair of particles 
in a specific kinematic configuration.

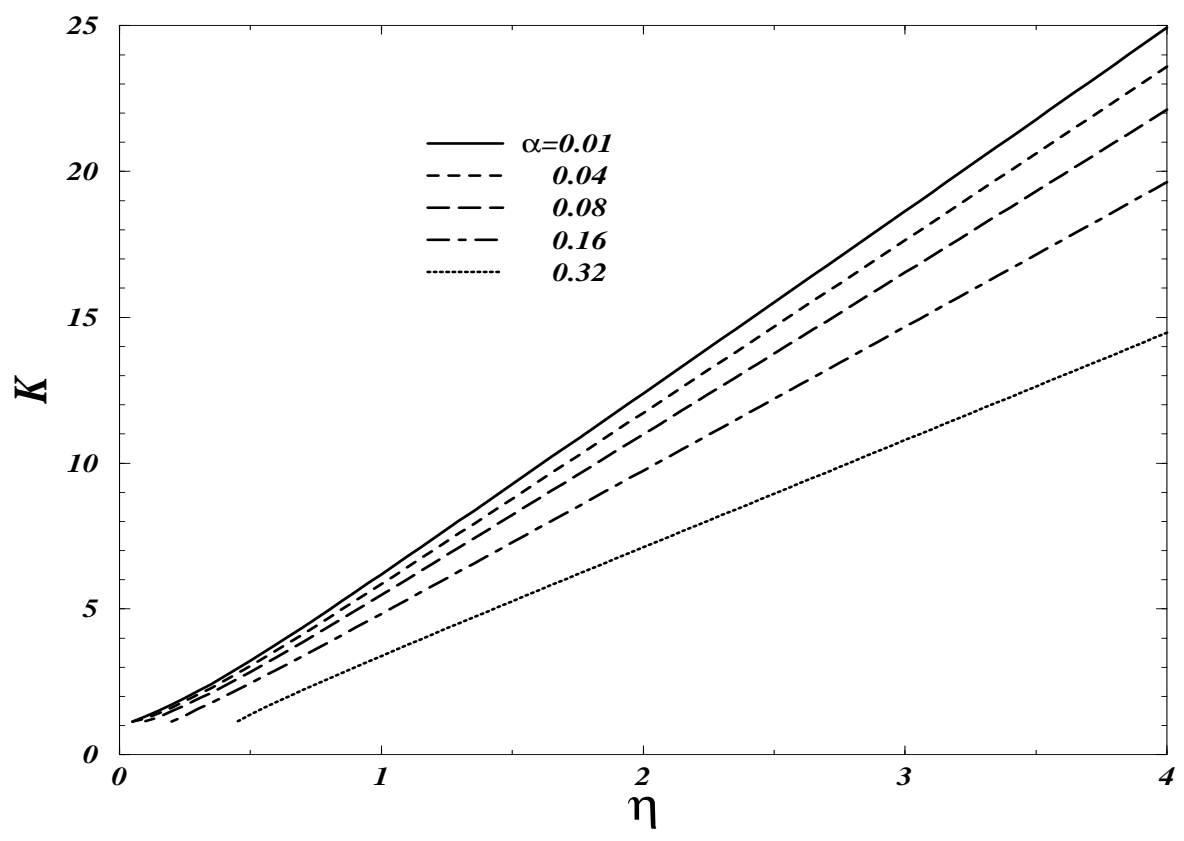

Fig. 2. The $K$-factor versus $\eta$ for various values of $\alpha$.

We show the behavior of the $K$-factor as a function of $\eta$ in Fig. 2 for various values of $\alpha$. The solid curve gives the $K$-factor for $\alpha=0.01$ and the dotted curve gives the $K$-factor for $\alpha=0.32$. For a fixed value of $\alpha$, the $K$-factor decreases as $\eta$ decreases. This is consistent with the expectation that the effects of the final-state interaction diminish as the velocity becomes relativistic. The limiting value is $K=1$ as $\eta=\alpha / v \rightarrow \alpha$. Figure 2 also shows that for a given value of $\eta=\alpha / v$, the $K$-factor decreases as $\alpha$ increases. It should be noted that the same value of $\eta$ corresponds to different velocities $v$ for different values of $\alpha$. To see the effect of final-state interaction as a function of $\alpha$ for a fixed value of $v$, we plot in Fig. 3 the $K$-factor as a function of $v$. As one observes, when the velocity is fixed, the $K$-factor increases as the coupling constant increases, indicating a greater effect of the final-state interaction as $\alpha$ increases. For all values of $\alpha$, the $K$-factor decreases with $v$ and goes to 
unity as $v$ approaches 1 . The decrease is very rapid for small values of $\alpha$.

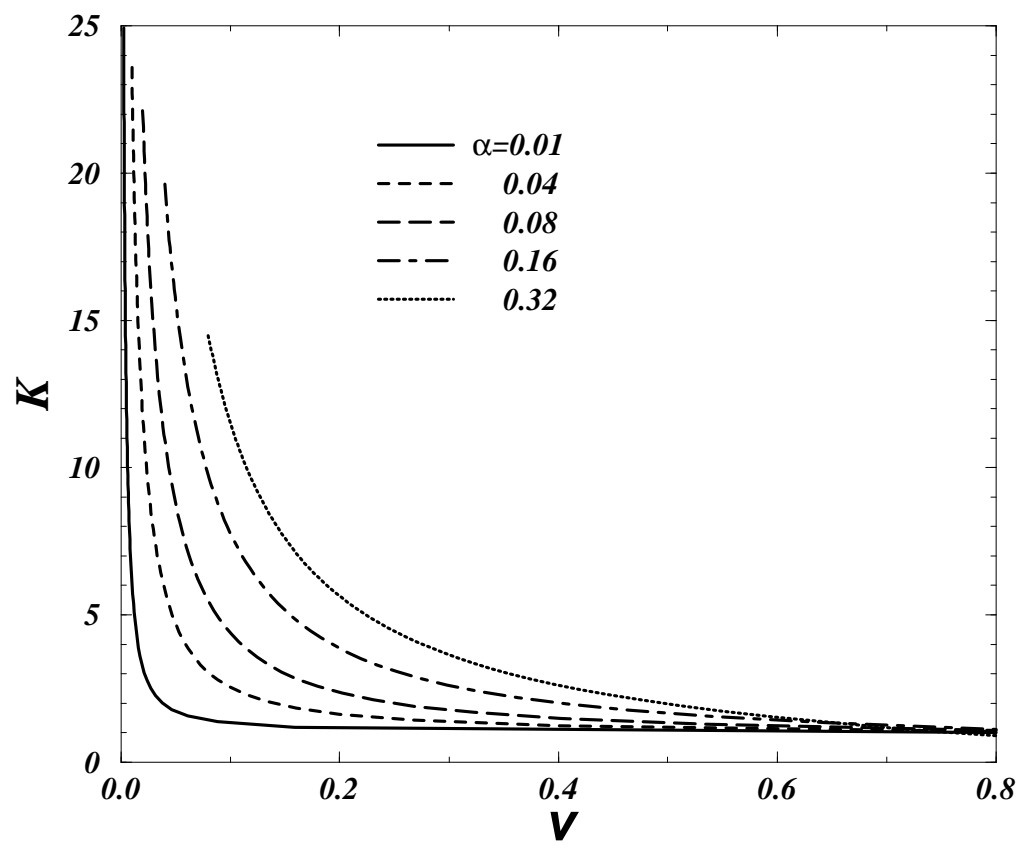

Fig. 3. The $K$-factor versus the velocity $v$ for various values of $\alpha$.

It is of interest to see how the $K$-factor obtained here is different from the Gamow factor in non-relativistic physics. In Fig. 4, we showed the ratio between the $K$-factor and the Gamow factor for various values of $\alpha$. As we expect, the ratio is almost 1 for weak coupling and the use of the Gamow factor is relatively safe there. However, if we increase $\alpha$ to 0.32 , the ratio decreases significantly. The Gamow factor overestimates the magnitude of the finalstate interaction. It cannot be used for the case with strong coupling. There is an effective screening of the long-range Coulomb interaction. As a consequence, the enhancement due to the long-range Coulomb-type interaction is reduced. It can also be observed in Fig. 4 that the ratio of $K / G(\eta)$ is a relatively slowly varying function of $\eta$ for $\eta>1$ but drops down rapidly as $\eta$ decreases in the region of small $\eta$.

It is worth pointing out that the expansion of $\mathcal{A}$ in Eqs. (34) and (41) is given as a series in powers of $p / \sqrt{\delta^{2}+k^{2}}$ which increases as the velocity $v$ increases. We still obtain convergent results for $v$ up to about 0.8 , but there is a limit on using such an expansion for greater velocities where $p / \sqrt{\delta^{2}+k^{2}}$ is too large to allow for a convergent term-by-term 
summation. A different expansion method is needed.

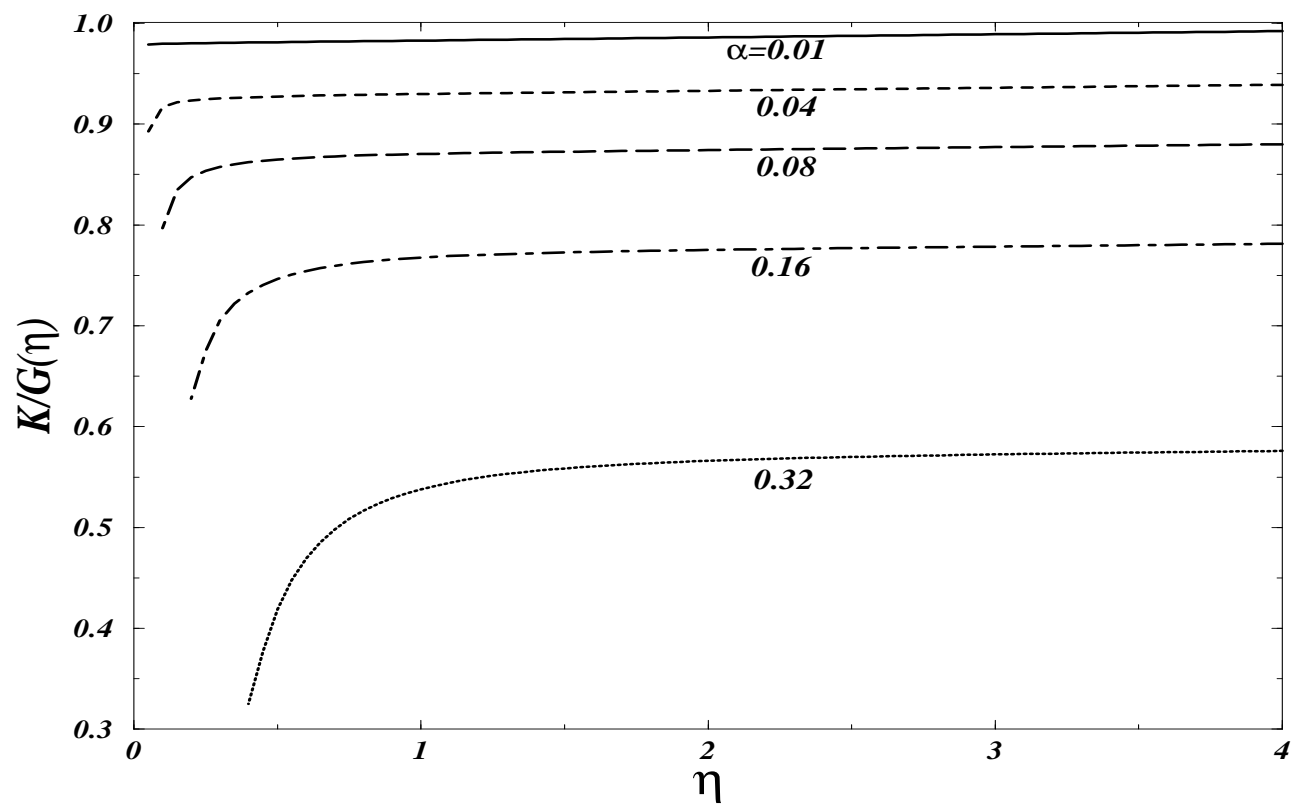

Fig. 4. The ratio between the $K$-factor and the Gamow factor $G(\eta)$ for various values of $\alpha$.

\section{CONCLUSIONS AND DISCUSSIONS}

The mutual final-state interaction between the produced particles has an effect on their rate of production. There will be similar effects if the particles interact via the initial-state interaction. The effects are simplest to be taken into account by using the method of the $K$-factor. One calculates the rate for the process as though there were no initial- or finalstate interactions, using, for example, the perturbation theory. The additional initial- or final-state interactions can be included by multiplying a $K$-factor as given by Eq. (8).

For Coulomb-type interactions, the $K$-factor has been traditionally taken to be the Gamow factor obtained as the absolute square of the wave function at the origin of the relative coordinate. With relativistic Coulomb wave functions, the wave function at the origin is infinite and the usual method is not applicable. The $K$-factor can be obtained as the overlap of the wave function with the Feynman amplitude.

Our investigation of the $K$-factor for the case of the production of a pair of scalar particles indicates that there are substantial deviations from the Gamow factor when the strength of the coupling is large. In particular, the proper treatment reduces the magnitude of the Gamow factor significantly. The reason for this reduction is that in the pair production, there is an effective screening of the Coulomb-type interaction arising from the effective "exchange" of one of the produced particles. 
We have presented an explicit formula for the relativistic modification of the Gamow factor for the production of a pair of bosons. Numerical results are also obtained to show the magnitude of the $K$-factor. The results of the $K$-factor can be applied to a class of processes in which the boson particles are produced and interacting with a Coulomb-type interaction.

The large modification of the Gamow factor for the production of two bosons studied here indicates the need to extend the present formalism to study the case of two fermions or two gluons. The application to fermions or gluons will be useful in the problem of production or reaction of quarks and gluons. As a pair of quarks or gluons interact with a strong colorCoulomb interaction with a coupling constant $\alpha$ about $0.2-0.4$, the simple results from the present study indicate that the effects of the initial- or final-state interaction for quark and gluon will be large, and the $K$-factor will be substantially different from what one obtains using the Gamow factor. We hope to study the modification of the Gamow factor for two produced fermions in our next investigation.

\section{ACKNOWLEDGMENTS}

Tha author would like to thank Dr. H. Crater for helpful discussions and valuable suggestions. This research was supported by the Division of Nuclear Physics, U.S.D.O.E. under Contract No. DE-AC05-96OR21400 managed by Lockheed Martin Energy Research Corp.

[1] H. A. Bethe and P. Morrison, Elementary Nuclear Theory, John Wiley and Sons, New York, 1956; J. M. Blatt and V. F. Weisskopf, Theoretical Nuclear Physics, John Wiley and Sons, New York, 1952, p. 731.

[2] G. Gamow, Zeit. Phys. 51, 204 (1928), see also L. I. Schiff, Quantum Mechanics, McGraw-Hill Company, 1955, p. 142.

[3] A. Sommerfeld, Atmobau und Spektralinien, Bd. 2. Braunschweig: Vieweg 1939

[4] R. M. Barnett, M. Dine, and L. McLerran, Phys. Rev. D22, 594 (1980).

[5] S. Güsken, J. H. Kühn, and P. M. Zerwas, Phys. Lett. 155B, 185 (1988).

[6] V. Fadin and V. Khoze, Soviet Jour. Nucl. Phys. 48, 487 (1988).

[7] V. Fadin, V. Khoze, and T. Sjöstrand, Zeit. Phys. C48, 613 (1990).

[8] S. J. Brodsky, A. H. Hoang, J. H. Kühn, and T. Teubner, Phys. Lett. B359, 355 (1995).

[9] L. Chatterjee and C. Y. Wong, Phys. Rev. C51, 2125 (1995).

[10] C. Y. Wong and L. Chatterjee, Proceedings of Strangeness '96 Meeting, Budapest, May 1996, ORNL-CTP-96-09 (hep-ph/9607316), published in Heavy Ion Phys. 4, 201 (1996).

[11] C. Y. Wong and L. Chatterjee, Z. Phys. C75, 523 (1997).

[12] I. T. Todorov, Phys. Rev. D3, 2351 (1971).

[13] H. W. Crater and P. Van Alstine, Ann. Phys. (N.Y.) 148, 57 (1983).

[14] H. W. Crater, R. Becker, C. Y. Wong and P. van Alstine, Phys. Rev. D46, 5117 (1992).

[15] L. D. Landau and E. M. Lifshitz, Quantum Mechanics, Pergamon Press, 1958, p. 118.

[16] M. E. Peskin and D. V. Schroeder, An Introduction to Quantum Field Theory, Addision Wesley Publishing Company, 1995. 
[17] H. W. Crater, Phys. Rev. A44, 7065 (1991).

[18] H. Crater has kindly pointed out to us that the model we are choosing for spinless QED neglects the effect of the transverse photons. If we include the effects of those photons then the two-body Klein Gordon equation has an additional repulsive term that makes the wave function vanish at the origin. See the paper of H. Crater and P. Van Altstine Phys. Rev. D30, 2585 (1984) Eq. 79 b.

[19] C. Y. Wong, hep-ph/9906291, (Physical Review D, in press). 